\title{
Comparison of Convolutional Neural Network Models for Recognizing Handwritten Letters
}

\author{
Author: Seojun Yang \\ E-mail: seojun.yang@concordacademy.org \\ <DOI:10.26821/IJSHRE.8.9.2020.8906 >
}

\begin{abstract}
We tried using mnst to recognize handwritten numbers, and We wanted to take it a step further and make a machine learning model that recognizes handwritten letters. There is a type of neural network called Convolutional Neural Network, or CNN. By using this neural network, we made a program that recognizes handwritten letters. We made a fully connected neural network without CNN, CNN without dropout and max pooling, and CNN with dropout and max pooling. We compared the efficiency of each model. After running each model, CNN with dropout and max pooling was more efficient in recognizing handwritten letters than the other 2 models since it prevented overfitting and had the highest test accuracy.
\end{abstract}

\section{INTRODUCTION}

\subsection{Design of the Research}

We are now living in the era of the fourth Industrial Revolution in which machine learning is one of the most-discussed technologies. Machine learning is a technique that creates an artificial neural network to predict the value of a certain model. Convolutional neural network, or $\mathrm{CNN}$, is an artificial neural network that is commonly used for classifying image data. The purpose of this research was to create $\mathrm{CNN}$ models that recognize handwritten letters with different numbers of layers and nodes to compare the performance of each model in terms of how much the difference in model affects the performance of the model. Furthermore, in order to enhance the performance of image-recognition, various techniques, such as dropout and max pooling, were applied to the CNN models.

\subsection{CNN (Convolutional Neural Network)}

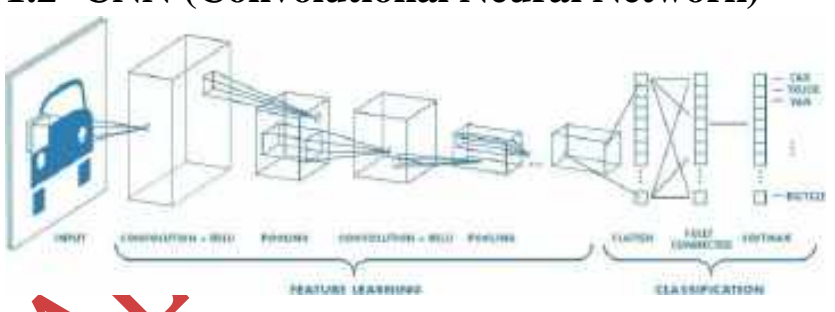

Fig 1: Convolutional Neural Network

Convolutional neural network, or $\mathrm{CNN}$, is a neural network that uses convolutional layers. Convolutional layers are layers of perceptrons that function as filters, or masks, that extract features from image data. In each cell of the masks, there is a weight assigned to it. CNN applies the mask on each pixel of the image. Then, CNN multiplies the greyscale value of each pixel by the weight of the mask's cell to get the feature of the image. However, since the mask can't get out of the image's cells, the cells in the extracted feature will be smaller than the original image. In order to extract a feature that is equal in size to the original image, we can put cells with zero weights, or paddings, around the original image. Furthermore, we can apply a technique called max pooling. Max pooling is pulling out maximum values of each chunk of the extracted feature. Max pooling helps prevent overfitting in that it gives a general sense of the feature rather than specific feature. Also, it decreases the number of cells in the extracted feature, which helps CNN decrease the number of operations to deal with the feature. Once CNN deals with the features, the convolutional layers or max pooling layers put their features into an activation function.

\subsection{Design of the Paper}

Section two is about data preprocessing. I loaded the csv file containing handwritten letters as a dataset. I scaled the dataset so that the values between 0 and 255 
Volume 8 Issue 9 September 2020

become values between 0 and 1 in grayscale. Section three is about concepts that affect efficiency of machine learning and three different results of learning. Finally, in the conclusion part, I put the results altogether.

\section{DATA PROCESSING}

We used a csv file from Kaggle for this research. Each cell in the csv file used in this research represents a pixel in an image of a handwritten letter in size $28 * 28$ pixels. Specifically, each cell contains the grayscale value of the pixel between 0 and 255 . The file also contains labels for each image.

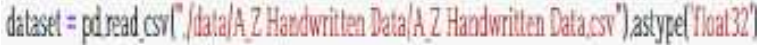

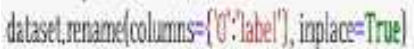

\section{Fig 2: Loading Dataset}

We loaded the csv file as float 32 .

\section{$X=$ dalaxtdropl'tabe', axis =11 \\ $Y=$ dalaset['label] \\ $X$ train, $X$ lest, $Y$ train, $Y$ test = traintest soitifX,Y \\ $X$ Lrain $=X$ train[:LCcco]

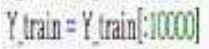 \\ $X|\mathrm{es}|=X \mid \mathrm{les}[15000)$ \\ $Y$ test $=Y$ lest 15000}

\section{Fig 3: Train Set and Test Set}

$\mathrm{X}$ represents attribute, and Y represents class. For the train set, we loaded 10,000 attributes and 10,000 classes. For the test set, we loaded 5,000 attributes and 5,000 classes. Train set is a set of features and labels that the model learns from. On the other hand, a test set is a set of features and labels that the model has not dealt with. Otherwise, overfitting can happen.

\section{Fistedodila \\ stardard sabre = Mrinlascaler||}

standerd sales:fin (X (train)

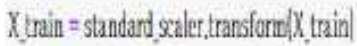 \\ $X$ lest $=$ sandard salet transform $(X$ lest $)$

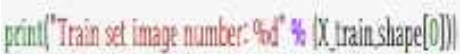

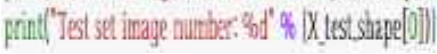

\section{Fig 4: Scaling}

We converted the grayscale values between 0 and 255 into decimals between 0 and 1 to reduce the size of the initial value of the dataset so that the size would not disproportionately increase during the process of machine learning to a point at which the calculation would take a large amount of time.

\section{print(Y Yest, Y Y train}

\section{pltt.igure $($ figsize $=(12,10))$ \\ row, colums $=4,4$ \\ for iin ange(16): \\ pitsabplatt(colums, [Ww, iti]

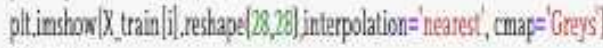 pllisworl)}

Fig 5: Printing Sample Handwritten Letters
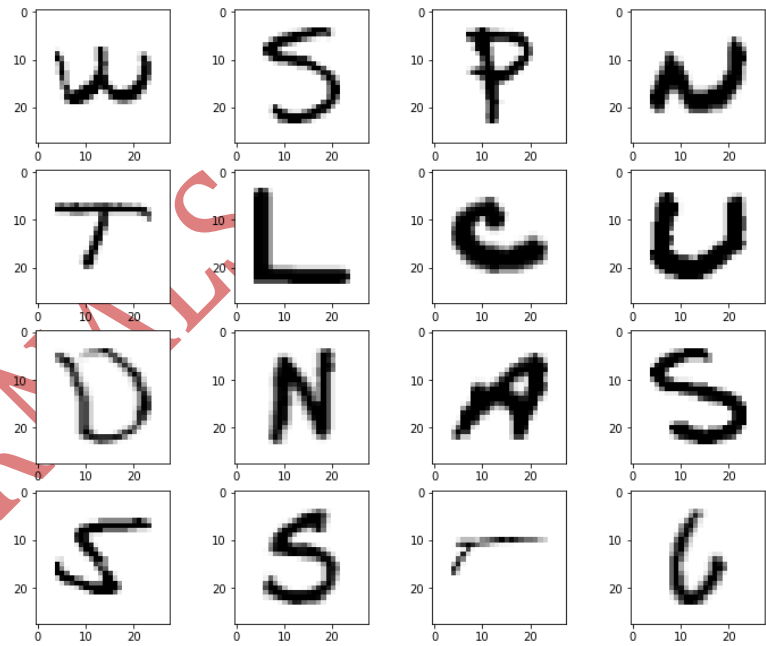

Fig 6: Sample Handwritten Letters

We can confirm that the dataset contains images of handwritten letters in size of $28 * 28$ pixels.

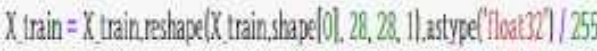

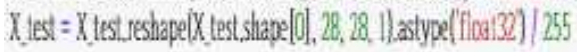

\section{Y. train $=$ ap utitis, to calegyrically train)

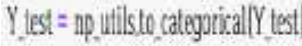

\section{Fig 7: Reshaping}

We converted the one dimensional dataset in the csv file that consists of 784 rows into two-dimensional partitions in size of $28 * 28$ pixels since we wanted to apply techniques that require referring to cells around a certain point in the image, such as max pooling.

\subsection{Overfitting}

Training the model for a long time does not benefit the accuracy of the model; doing so increases the risk of overfitting. Overfitting causes the model to output 
Volume 8 Issue 9 September 2020

highly accurate results when it deals with the data in the train set, while the model outputs relatively inaccurate results when it deals with data that are not in the train set. For instance, in the graph below, the red line drawn by the model perfectly divides the red circles and the blue circles. In other words, the model, which distinguishes between red circles and blue circles, would output highly accurate results when it is given the data of all the circles in the graph below.

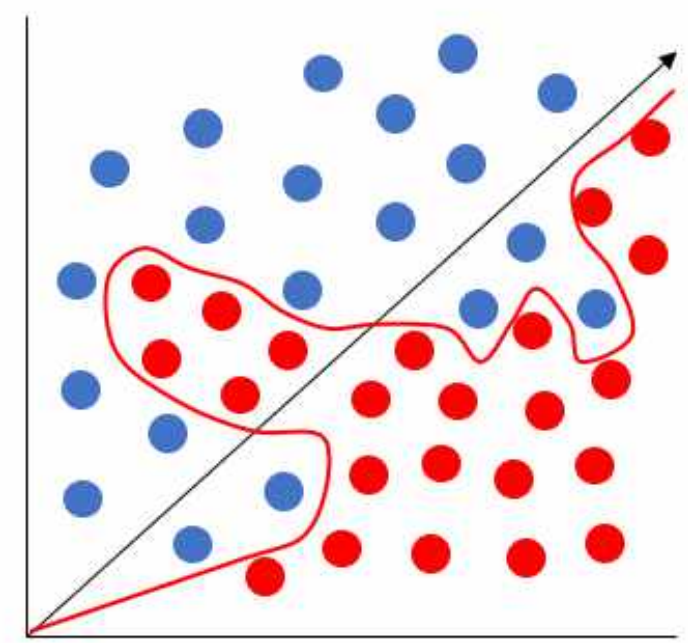

Fig 8: Overfitting

On the other hand, if the model is given data that are not in the graph above, then the red line will not represent the characteristics of the data accurately. In fact, the black line represents the characteristics of data better than the red line does. Overfitting, such as the one shown in the example, can happen when there are too many layers of perceptrons, when the learning process takes too long, and when the train set and test set do not differ,

There are two main ways of preventing overfitting: early stopping and dropout. At some point during the learning process of the model, the accuracy of the model in dealing with the train set will increase, while its accuracy in dealing with the test set decreases. Early stopping stops the learning process as soon as the epoch reaches the point. As aforementioned, having too many layers of perceptrons can cause overfitting. Dropout randomly picks several perceptrons in each epoch and inactivates them to avoid such cause of overfitting. Specifically, dropout prevents the model from being biased.

\subsection{One Hot Encoding}

Categorical data consists of labeled data. However, the activation functions of the perceptrons need the data to be numeric values to produce a reasonable output. One hot encoding is converting the labeled data into numeric data. When categorical data is not converted into the numeric form, there is a risk of the model misinterpreting the data and giving unreasonable meanings to the data. For instance, if a model generates 4.5 as output, then the category of the output cannot be identified since 4.5 is neither 4 nor 5 .

\subsection{Max Pooling}

Max pooling is a technique that takes maximum value from each region of the data. By doing so, we are able to compress information, and thus make the process of machine learning faster. Since max pooling allows the model to deal with maximum values that represent the whole dataset instead of every single value of the dataset, we can expedite the process.

\subsection{Drop Out}

Since training the model for a long time increases the risk of overfitting, we used a technique called dropout, which randomly drops out nodes during training.

\section{EXPERIMENTS}
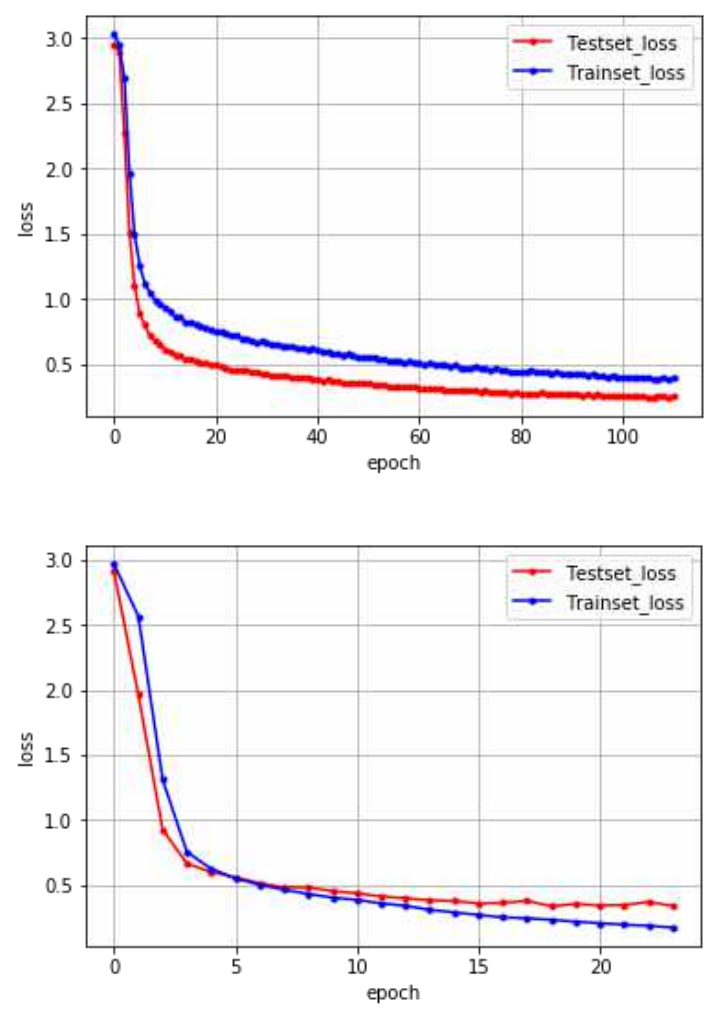
Volume 8 Issue 9 September 2020

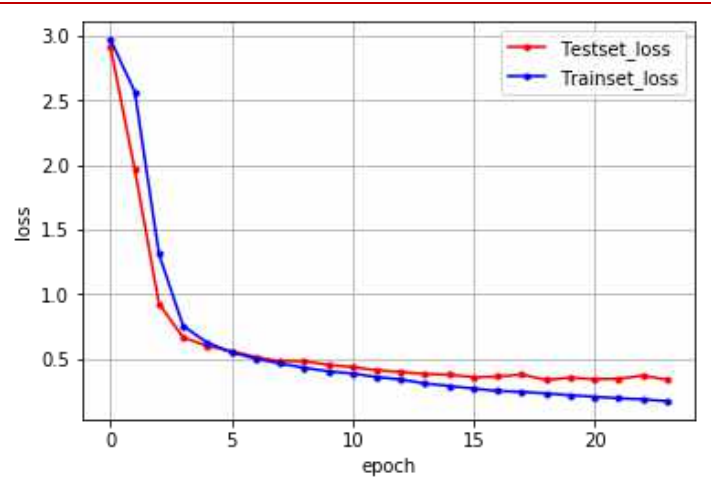

Fig 9: Error Loss Graph of Fully Connected Neural Network without CNN, CNN without Dropout and Max Pooling, CNN with Dropout and Max Pooling

\begin{tabular}{|c|c|c|c|}
\hline & $\begin{array}{c}\text { Fully } \\
\text { Connecte } \\
\text { d Neural } \\
\text { Network } \\
\text { without } \\
\text { CNN }\end{array}$ & $\begin{array}{c}\text { CNN } \\
\text { without } \\
\text { Dropout } \\
\text { and Max } \\
\text { Pooling }\end{array}$ & $\begin{array}{c}\text { CNN with } \\
\text { Dropout } \\
\text { and Max } \\
\text { Pooling }\end{array}$ \\
\hline $\begin{array}{c}\text { Final Epoch } \\
\text { (max 200) }\end{array}$ & 200 & 24 & 111 \\
\hline Accuracy & 0.8608 & 0.9152 & 0.9340 \\
\hline
\end{tabular}

Table 1: Final Epoch and Accuracy of Each Model

Fully connected neural network without CNN took the maximum epoch since CNN wasn't applied. Even after 200 epochs, the model didn't converge enough and had a test accuracy of 0.8608 , which was the lowest of all three models. On the other hand, CNN without dropout max pooling ended after only 24 epochs, which is much less than what the fully connected neural network without CNN took. Also, the test accuracy of $\mathrm{CNN}$ without dropout and max pooling is higher than the fully connected neural network without CNN. The reason for this improvement in performance was the use of CNN. Finally, CNN with dropout and max pooling ended after 111 epoch, which is more than what CNN without dropout and max pooling took. However, $\mathrm{CNN}$ with dropout and max pooling used the epoch to prevent overfitting, which ended up giving a test accuracy of 0.9340 , the highest of all three models.

\section{CONCLUSION}

We wanted to know how much of a difference making layers and using preprocessing techniques make in terms of efficiency of Convolutional Neural Network that recognizes handwritten letters. The techniques we used included max pooling and drop out, which expedite the learning process and prevent overfitting. On the other hand, we also created another model that we didn't apply the layers and techniques to. We compared the efficiency of the two models to see the difference that the layers and techniques make.

CNN with dropout and max pooling was the model with highest accuracy. It took 111 epochs before early stopping. CNN without dropout and max pooling was the model with lowest final epoch. Fully connected neural network without $\mathrm{CNN}$ was the model with lowest accuracy. The model took maximum final epoch.

From the results above, we can conclude that CNN with dropout and max pooling was the best model for recognizing handwritten letters in terms of accuracy. Dropout prevented overfitting, which increased the model's accuracy. Max pooling also helped the model converge faster.

\section{REFERENCES}

[1] Kaggle "AZ Handwritten Alphabets"

https://www.kaggle.com/sachinpatel21/azhandwritten-alphabets-in-csv-format

[2] Structure of Convolutional Neural Network

https://divamgupta.com/assets/images/posts/imgseg/im age1.png?style=centerme

[3] Deep Learning for Everyone, Taeho Cho, 2017 ISBN 979-11-6050-371-5 